\title{
FEM SIMULATION OF THE NANOINDENTATION TEST WITH RIGID AND NON-RIGID INDENTER
}

\begin{abstract}
J. Kováŕr ${ }^{*}$ V. Fuis ${ }^{* *}$
Abstract: This paper focuses on the comparing of the results of the FEM simulations of fused silica nanoindentation tests. Fused silica is modelled like bilinear elasto-plastic material with the parameters estimated from the experiment. FEM simulations are made with the $2 D$ axisymmetric conical indenter which is equivalent to the Berkovich indenter and the indentation curves are evaluated. The FEM simulations were made with the rigid and the non-rigid indenter and the difference between the indentation curves obtained with these models is assessed. The evaluated indentation curves are compared with the indentation curves obtained from the experiment.
\end{abstract}

\section{Keywords: Nanoindentation, Conical indenter, Silica, FEM}

\section{Introduction}

The nanoindentation test is the test in which the material parameters are measured at low values of the indenter displacement. The diamond indenter is pressed into the tested material and the displacement of the indenter is measured. The values of the penetration are in the order of nanometres or few micrometres. This allows to use nanoindentation test for measurement of the material parameters that cannot be measured with the conventional tests.

Nanoindentation test can be used for measurement of the material parameters of the surfaces, thin layers (Bull, 2005), local parameters or the parameters of the plastic behaviour (Torres-Torres et al., 2010). The FEM simulation of the nanoindentation test will be used for determination of the plastic properties of fused silica in this paper.

\section{Theory}

\subsection{Fused silica plasticity}

The yield stress of fused silica is much higher than the tensile or compressive strength. This causes that the fused silica fractures before the start of the plastic behaviour if the conventional tests are used. If the nanoindentation test is used, the volume with high stresses is very small. This causes that the brittle fracture will not be initialized. Then higher values of the stresses can be reached and plasticity occurs. Then, opposite to the conventional tests, the nanoindentation can be used for determination of the parameters of the fused silica plasticity.

According to the Oliver-Pharr analysis (Oliver, 1992), the modulus of elasticity and hardness can be directly determined from the nanoindentation test and another material parameters can be determined from the inverse FEM calculation.

Ing. Jaroslav Kovár̆: Institute of Solid Mechanics, Mechatronics and Biomechanics, Brno University of Technology, Technická 2896/2; 616 69, Brno; CZ, Jaroslav.Kovar@vut.cz

** Assoc. prof. Ing. Vladimír Fuis, Ph.D.: Centre of Mechatronics - Institute of Thermomechanics of the Czech Academy of Sciences - branch Brno and Faculty of Mechanical Engineering, Brno University of Technology, Technická 2896/2; 619 69, Brno, CZ, fuis@it.cas.cz 


\subsection{FEM simulation of the nanoindentation test}

The dependency of the resultant force on the displacement of the indenter, called indentation curve, is measured during the nanoindentation test. The stress-strain curve is more important for the description of the material. This curve can be determined by the FEM. The inverse FEM method is used for these calculations.

Firstly the suitable material model is chosen and the first estimation of the parameters of this model is set. Then the FEM simulation of the nanoindentation is done. The result of this calculation is indentation curve. The evaluated indentation curve is compared with the indentation curve from the experiment.

According to the differences between these curves, new parameters of the model of material are set for next iteration. This calculation is repeated until the difference between the experimental and the evaluated indentation curves is not smaller than allowed value. Then the parameters of the model of material used for the last FEM calculation are the parameters of the model of tested material.

This procedure is mostly used for the determination of the material properties from the FEM calculation. The biggest problem of this method is that many of FEM simulations need to be done for evaluating of the parameters of the material.

\subsection{Indenters which are mostly used for nanoindentation}

Many indenters can be used for nanoindentation test. The often used indenters are shown in Fig. 1. The most used indenter is the Berkovich indenter. Another often used indenters are the Vickers indenter, the cube corner indenter, the spherical indenter, the conical indenter and the flat punch indenter.

The biggest advantage of the spherical indenter is that the tip of this indenter is not sharp. When the sharp indenter is used, the elasto-plastic deformations occur nearly at the start of the indentation. When the spherical indenter is used, the part of the elastic and the part of the elasto-plastic behavior can be distinguished at the indentation curve (Park, 2004).

When the flat punch indenter is used, the unloading curve is straight because the unloading phase is only elastic (Guillonneau, 2012). The Berkovich and the Vickers indenters have similar properties, but the tip of the Vickers indenter is sharper. The sharpest tip have the cube corner indenter, whose tip of the indenter is the corner of the cube.
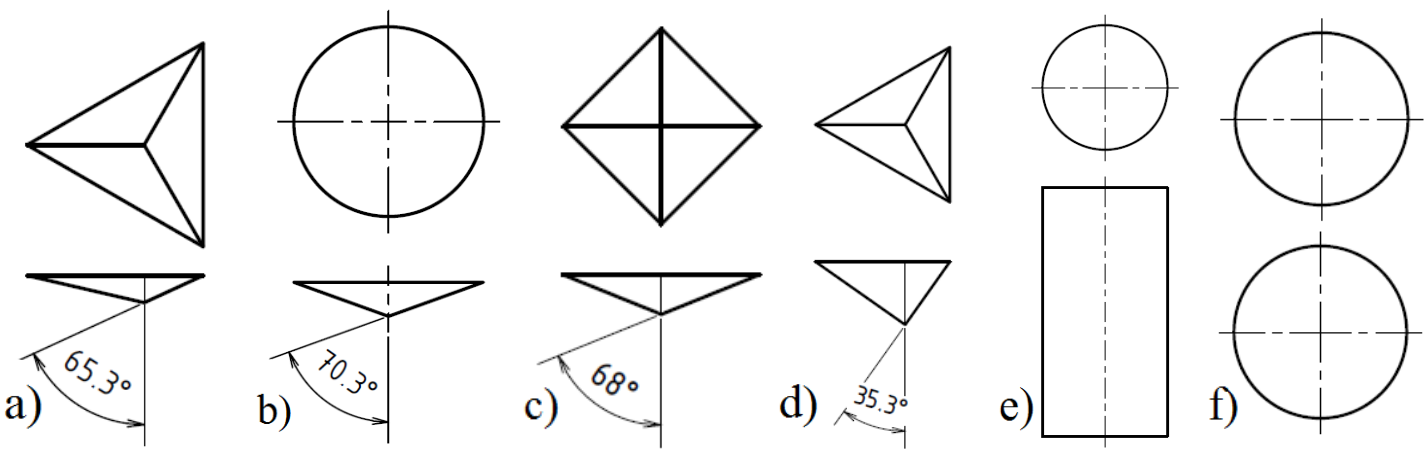

Fig. 1: Indenters: a) Berkovich, b) conical, c) Vickers, d) cube corner, e) flat punch, f) spherical

\subsection{Berkovich indenter}

The Berkovich indenter was assumed for the FEM calculations. This indenter was firstly designed with the angle of the tip $65.03^{\circ}$. This angle was designed to make the surface area of the Berkovich indenter the same as the surface area of the Vickers indenter. The mostly used Berkovich indenter for nanoindentation have the angle $65.27^{\circ}$ (Fig. 1a). This indenter has the same projected area to the plane perpendicular to the axis of the indenter as the Vickers indenter (Fischer-Cripps, 2002). The projected area is used for determination of the modulus of elasticity from measured data.

The same projected area like Berkovich indenter has a conical indenter with semi-angle $70.3^{\circ}$ (Fig. $1 \mathrm{~b}$ ). This approximation of the shape of the Berkovich indenter was firstly used for determination of the contact pressure at the top of the Berkovich indenter but this model is still used for modelling of the nanoindentation test with FEM because it has the same relation between the depth of penetration and the contact area like Berkovich indenter (Fischer-Cripps, 2002). 
Berkovich indenter is usually made of diamond, which has a high value of the modulus of elasticity (1 $141 \mathrm{GPa}$ ), therefore it can be modelled like rigid body. Even though the elastic deformation of the indenter can influence the results because the displacements of the indenter are in the order of nanometres and the contact pressure is high. In the real applications there can be bluntness of the Berkovich indenter which has big impact on the results (Torres-Torres et. al., 2010). Sharp indenter will be assessed for calculations in this paper.

\section{FEM model of axisymmetric conical indenter}

This task was modelled like contact axisymmetric task because the conical indenter was assumed for this calculations. The topology and the mesh is shown in Fig. 2. The PLANE 182 elements were used. The mesh was refined under the tip of the indenter where the size of the edge of the elements near the contact area was $35 \mathrm{~nm}$. The contact elements CONTA 172/ TARGE 169 were used in the contact area.

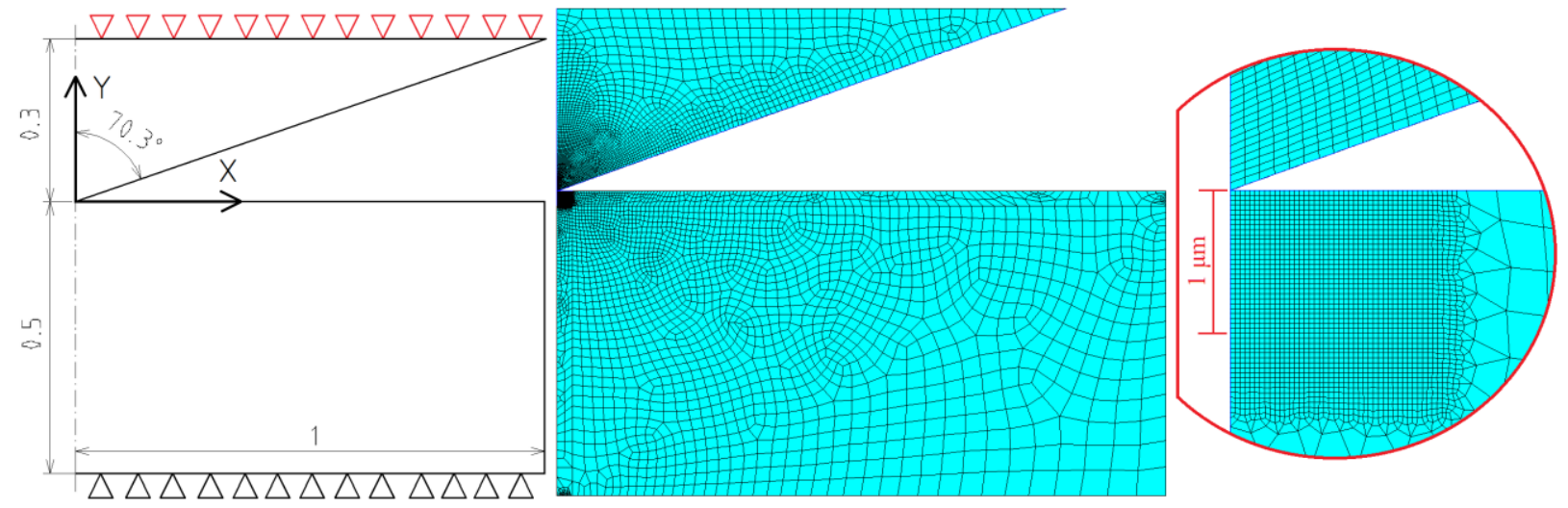

Fig. 2: The geometry and the mesh of the axisymmetric model

The diamond indenter was firstly modelled like rigid and then like linear elastic material with $E=1141 \mathrm{GPa}$ and $\mu=0.07$ (Knapp et. al., 1999). The tested specimen was assumed from the fused silica, modelled with bilinear model of material with elastic parameters $E=72 \mathrm{GPa}, \mu=0.17$, which were taken from (Torres-Torres et al., 2010), and parameters $R_{p}$ (yield strength) and $E_{t}$ (tangent modulus), which are describing the plastic behaviour of the material. The sensitivity analyses of the indentation curve to the $R_{p}$ and $E_{t}$ were made and with help of them the values of these parameters were estimated.

The specimen was loaded by the vertical displacement of the cone. The displacement of $1340 \mathrm{~nm}$ against the way of the $\mathrm{Y}$ axis was applied. The vertical displacement of the lower line of the specimen was fixed. Other boundary conditions were made from the axisymmetry of the specimen.

\section{Results}

The results of the computational modelling are the indentation curves for different values of the $R_{p}$ and $E_{t}$. Fig. 3 shows that in the case with the rigid conical indenter, the calculated indentation curve perfectly fits the curve from the experiment in the loading phase. When unloading occurs, the evaluated unloading curve is steeper than unloading curve from the experiment. Part of this error is caused by modelling the indenter like rigid. According to the Oliver-Pharr analysis (Oliver, 1992) the slope of the unloading curve was used to determine the reduced modulus. This modulus is not the same as in the case of the experiment because it is influenced by the elastic properties of the measured material and the indenter, which is perfectly rigid here. When the correction to the modulus of elasticity of the material was done, this model still showed higher value of the modulus of elasticity than was given into the calculation (74.8 GPa, relative error is $4 \%$ ).

In the case with the non-rigid conical indenter, the indentation curve shows lower values of the forces because there is elastic deformation of the tip of the indenter. The yield stress was increased and then evaluated indentation curve perfectly fits the curve from the experiment. The elastic modulus evaluated from this curve is near to the elastic modulus which was assumed. Assumed elastic modulus was $72 \mathrm{GPa}$ and evaluated is $73.1 \mathrm{GPa}$. Relative error is $1.5 \%$ in this case. 


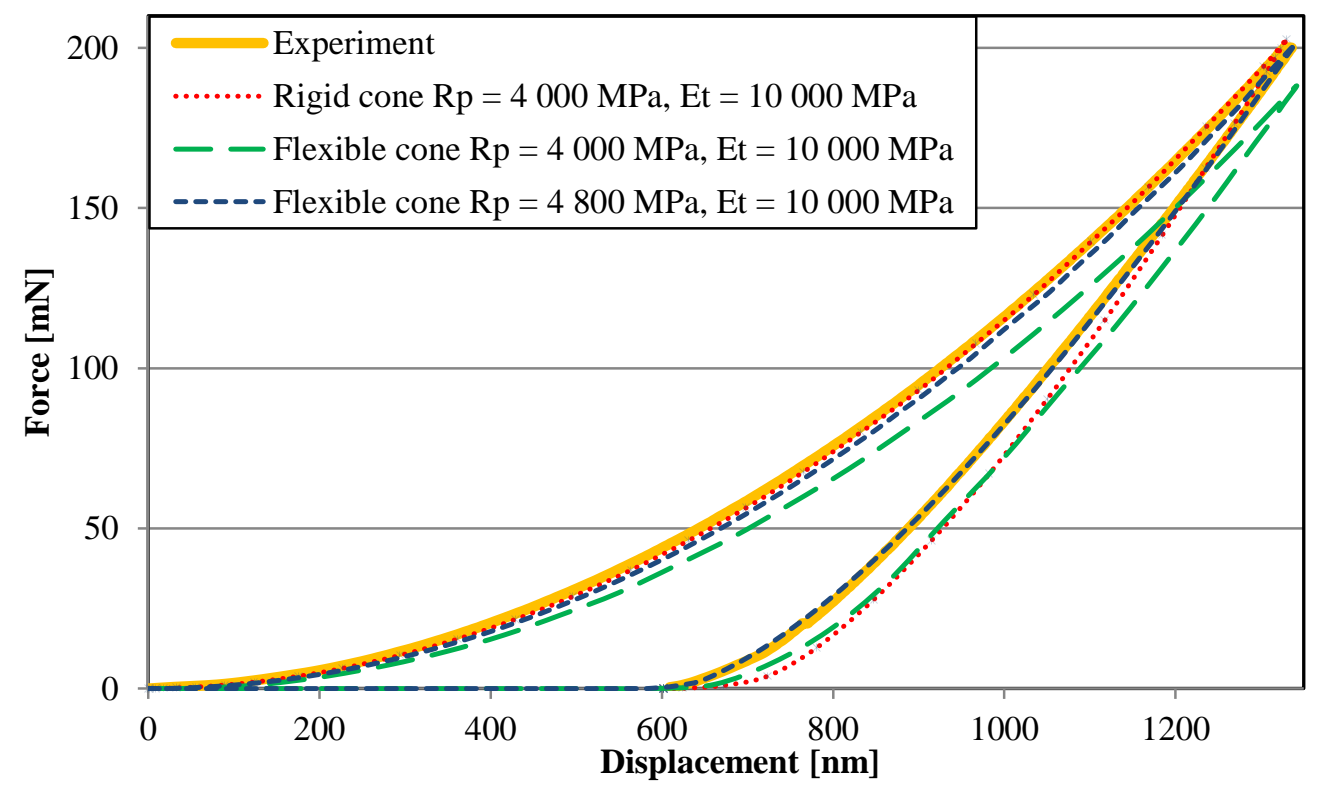

Fig. 3: Comparing the indentation curves for conical indenter

\section{Conclusions}

The nanoindentation test is the test in which the depths of the penetration are small. It can be used for determination of the fused silica plasticity which cannot be evaluated by the conventional tests because the glass fails by the brittle fracture.

Nanoindentation test of the fused silica was modelled. The evaluated indentation curves calculated with the conical rigid and non-rigid indenter were compared with the experimental data. This indenter is equivalent to the Berkovich indenter. The results with the rigid indenter show slightly higher values of the resultant forces and steeper unloading curve than experimental data. It is partially caused by the model of the rigid indenter which influences the relative modulus.

Results with the non-rigid conical indenter are more accurate. The yield stress was increased to the $4800 \mathrm{MPa}$, because the indentation curve showed lower values than in the previous case. Than the calculated indentation curve perfectly fits to the curve from the experiment.

\section{Acknowledgement}

The experimental data were measured by the Institute of Physics of the Academy of Sciences of the Czech Republic, Joint Laboratory of Optics of Palacky University and Institute of Physics AS CR.

This study was realized with the support by the grant FSI-S-17-4386 and with the institutional support RVO: 61388998.

\section{References}

Bull, S. J. (2005). Nanoindentation of coatings. Journal of Physics D: Applied Physics, 38, 24, R393-R413.

Fischer-Cripps, A. C. (2002). Nanoindentation Testing. Nanoindentation, pp 20-35.

Guillonneau, G., Kermouche, G., Bec, S., \& Loubet, J.-L. (2012). Determination of mechanical properties by nanoindentation independently of indentation depth measurement. Journal of Materials Research, 27, pp 25512560.

Knapp, J. A., Follstaedt, D. M., Myers, S. M., Barbour, J. C., \& Friedmann, T. A. (1999). Finite-element modeling of nanoindentation. Journal of Applied Physics, 85, 3, pp 1460-1474.

Oliver, W. C., \& Pharr, G. M. (1992). An improved technique for determining hardness and elastic modulus using load and displacement sensing indentation experiments. Journal of Materials Research, 7(06), pp 1564-1583.

Park, Y. J., \& Pharr, G. M. (2004). Nanoindentation with spherical indenters: finite element studies of deformation in the elastic-plastic transition regime. Thin Solid Films, 447-448, pp 246-250.

Torres-Torres, D., Muñoz-Saldaña, J., Gutierrez-Ladron-de Guevara, L. A., Hurtado-Macías, A., \& Swain, M. V. (2010). Geometry and bluntness tip effects on elastic-plastic behaviour during nanoindentation of fused silica: experimental and FE simulation. Modelling and Simulation in Materials Science and Engineering, 18(7). 